\title{
Premier congrès de la Société internationale francophone d'éducation médicale : bilan et perspectives
}

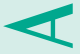

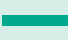
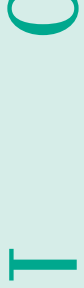

Le premier congrès de la Société internationale francophone d'éducation médicale (SIFEM), consacré à la problématique de "l'évaluation en éducation des sciences de la santé : une réponse aux enjeux de la santé" s'est tenu à Beyrouth les $1^{\text {er }}$ et 2 juin 2006 sur le campus des sciences médicales de l'Université Saint-Joseph, précédé les 30 et 31 mai d'ateliers de formation. Cent soixante enseignants venant de différents pays de la francophonie - France, Canada, Belgique, Suisse, Tunisie, Maroc, Côte d'Tvoire, Sénégal, Guinée et Liban - ont participé à cette manifestation, apportant et échangeant leur savoir, leur expertise et leur enthousiasme. De l'avis unanime, ce congrès a répondu aux attentes; il marque ainsi une étape importante et fondatrice dans la vie de notre jeune société, la SIFEM, nouvelle venue dans le concert des institutions savantes dédiées à la pédagogie médicale. Au terme de cette étape, il nous parait utile d'analyser les facteurs qui ont assuré la réussite de ce congrès et de rappeler les perspectives qui doivent guider les orientations futures de la SIFEM.
\end{abstract}

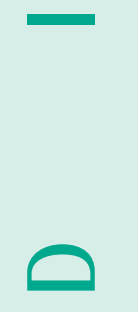

Quatre facteurs, nous semble-t-il, ont contribué à assurer le bon déroulement et le succès de ce congrès.

Le contenu scientifique et la pertinence thématique : l'évaluation des apprentissages et des enseignements, la recherche et les nouvelles technologies en éducation des sciences de la santé ont été les trois thèmes abordés et traités au cours de ce premier congrès. Ils sont actuellement au coour de la réflexion concer- nant tout système d'éducation, compte tenu de leur importance, de leur actualité et de la nécessaire créativité, indispensable à l'adaptation continue et à l'amélioration constante des pratiques en cours.

La remarquable prestation de la plupart des intervenants, au cours des séances plénières, des 12 ateliers-débat, des 52 communications orales, des 8 communications affichées et plus particulièrement celle des trois grands conférenciers ont permis de porter un regard original sur l'éducation médicale, empreint d'un recul fécond: Jacques Roland, Charles Boelen, Michel Serres ont tour à tour rappelé la nécessaire réflexion sur les changements survenus dans l'environnement médical et les moyens de s'y adapter, l'importance d'une éducation médicale à l'écoute des besoins d'une population et l'indispensable éveil des médecins à l'aspect humaniste de leur exercice. Ces conférences seront rapportées dans les numéros successifs de Pédagogie Médicale

La qualité de l'organisation, où tout a été mis en cuvre pour assurer le meilleur quant à l'accueil, les différents déplacements, la répartition des salles, les pauses, la transmission en direct sur le site de l'Université médicale virtuelle francophone (UMVF) (www.canal-u.education.fr) et toute l'infrastructure qui a fait en sorte que tout se déroule dans la fluidité, sans accroc, sans hésitation.

L'ambiance régnante, favorisée par la par- 
ticipation sans réserve des responsables de nos institutions partenaires, le Forum international francophone de pédagogie médicale du Québec et le Conseil pédagogique de la Conférence internationale des doyens et des facultés de médecine d'expression française (CIDMEF). Caractérisée par une convivialité généreuse, par la qualité des échanges humains, par une grande chaleur humaine, par la joie de se retrouver et de partager un moment fort de la vie de la pédagogie médicale francophone, elle a connu son point d'orgue lors du dîner de gala du dernier soir, véritable fête de clôture.

La SIFEM a acquis droit de cité. Il lui reste à maintenir son élan et suivre les orientations suivantes :

Poursuivre la coopération étroite avec les deux grandes institutions francophones sours, citées plus haut, avec lesquelles un accord est intervenu pour que chacune d'entre elles organise, à tour de rôle et tous les trois ans, sa propre manifestation internationale, à l'occasion de laquelle, respectivement, chacune des autres est invitée à y tenir une session spécifique, comme cela a été le cas à Cotonou en 2005 et à Beyrouth en 2006 et comme cela le sera en 2007 à Mont Tremblant (Québec), lors de la prochaine réunion du Forum. Mais il ne s'agit là que d'activités ponctuelles, qui ne sauraient répondre à l'essentiel des missions de la SIFEM.

Entreprendre un travail de fond, continu, qui fasse le socle de la vie de la SIFEM, est ainsi nécessaire pour répondre au mandat qu'elle s'est donnée conformément à ses objectifs : "dynamiser la recherche sur les fondements et la philosophie de l'éducation médicale, sur les rouages et les mécanismes psychologiques qui mènent à la connaissance et à la compétence, sur la mise au point de démarches et processus d'enseignement, d'apprentissage, d'évaluation et ce, tout au long du continuum de l'éducation médicale ". C'est dans cette perspective qu'ont été lancées les actions prioritaires qui connaissent actuellement un début de mise en place et qui donneront véritablement sens à la présence de notre société. Ces actions doivent se faire, bien sûr, dans le respect de la spécificité, de la légitimité individuelle et des missions respectives de chacune des institutions "soeurs" et plus particulièrement du conseil pédagogique de la CIDMEF.

Contribuer à la promotion, à la reconnaissance et à la diffusion de la revue Pédagogie Médicale, qui est l'organe officiel d'expression de notre société et dont le succès grandissant fait honneur à tous ceux qui se dévouent pour en garantir la parution : la maison d'édition Acalis bien sûr, mais aussi et surtout Jean Jouquan, rédacteur en chef, sur qui, après Bernard Charlin, repose la lourde tâche de veiller à la qualité des contenus et d'assurer une diffusion régulière.

La SIFEM est née en avril 2003, à Nancy, lors des $X V^{e s}$ Journées internationales francophones de pédagogie médicale, après plusieurs années de concertation et de préparation. Il fallait le courage et la foi de quelques pionniers pour croire en la nécessite de cette création et accepter l'aventure. Le pari est gagné; le congrès de Beyrouth en est le témoignage.

Professeur Pierre Farah, doyen honoraire, Président de la SIFEM mailto:pfarah@usj.edu.lb 